\title{
The 3D Kuramoto-Sivashinsky Equation for Nonequilibrium Defects Interacting through Self-Consisting Strain and Nanostructuring of Solids
}

\author{
V. I. Emel'yanov \\ Physics Faculty, Lomonosov Moscow State University, Moscow 119991, Russia \\ Correspondence should be addressed to V. I. Emel’yanov; emelyanov.vladimir@gmail.com
}

Received 25 June 2013; Accepted 2 September 2013

Academic Editors: R. Hong, G. Jin, and A. Pyatenko

Copyright (c) 2013 V. I. Emel'yanov. This is an open access article distributed under the Creative Commons Attribution License, which permits unrestricted use, distribution, and reproduction in any medium, provided the original work is properly cited.

It is shown that the bulk defect-deformational (DD) nanostructuring of isotropic solids can be described by a closed threedimensional (3D) nonlinear DD equation of the Kuramoto-Sivashinsry (KS) type for the nonequilibrium defect concentration, derived here in the framework of the nonlocal elasticity theory (NET). The solution to the linearized DDKS equation describes the threshold appearance of the periodic self-consistent strain modulation accompanied by the simultaneous formation of defect piles at extremes of the strain. The period and growth rate of DD nanostructure are determined. Based on the obtained results, a novel mechanism of nanostructuring of solids under the severe plastic deformation (SPD), stressing the role of defects generation and selforganization, described by the DDKS, is proposed. Theoretical dependencies of nanograin size on temperature and shear strain reproduce well corresponding critical dependencies obtained in experiments on nanostructuring of metals under the SPD, including the effect of saturation of nanofragmentation. The scaling parameter of the NET is estimated and shown to determine the limiting small grain size.

\section{Introduction}

In recent years, the spontaneous formation of periodic nanostructures upon the energy input into solids (laser or particle beams, severe plastic deformation, electrochemical etching, and others) has become the subject of intensive studies. An effective tool for the theoretical investigation of this phenomenon is the nonlinear Kuramoto-Sivashinsky (KS) equation $[1,2]$. 2D KS equations with specific variables are used, for example, for the description of nanostructuring of solid surfaces using ion beams [3] and electrochemical etching [4]. Recently, a novel 2D KS equation has been derived for a defect-enriched solid layer formed at the surface by laser irradiation, and its numerical solutions have been used for the interpretation of the formation of surface nanoand microstructures under the action of laser pulses [5].

In [6], a model of the defect-deformational (DD) instability in the system of mobile defects (interstitials and vacancies), interacting in the bulk of an isotropic solid via quasistatic self-consistent strain, has been developed.
The mechanism of the bulk DD instability consists in the following. An initial fluctuation of the defect concentration gives rise to self-consistent strain. This is accompanied by the strain-induced defect fluxes enhancing initial defect concentration fluctuation while the diffusion tends to smooth it out. At exceeding of a certain critical defect concentration, the positive feedback leads to the onset of DD instability with arising of nanometer scale bulk strain modulations and piling of defects at extrema of the strain. We note that, although the consideration of [6] was focused on point defects, starting DD equations possess a generality enabling description of the DD selforganization of any dilatation centers (including small dislocation loops) undergoing the diffusion. (The diffusion of nanometer size dislocation loops was observed in [7].)

In this work, we show that, invoking the nonlocal stress concept of the nonlocal elasticity theory (NET) $[8,9]$, one can, under some restrictions, reduce the equations of the bulk DD self-organization to a 3D nonlinear partial differential equation for the defect concentration of the KS equation type. In the mean field approximation (MFA), the obtained DDKS 
equation is reduced to the KS equation with critical behavior, which describes the threshold self-organization of bulk DD nanostructures. The period of self-organized DD structure is shown to be proportional to the scaling parameter of the NET.

In this work, we confine to investigation of linear solutions to the obtained nonlinear isotropic DDKS equation. The threshold defect concentration, the growth rate, and the period of the DD structure formed are determined.

Based on obtained results, a novel mechanism of nanostructuring under the SPD, stressing the role of defects generation and self-organization, described by the DDKS, is proposed. We show that solutions of the DDKS equation describe accurately experimental critical dependencies of the mean grain size on temperature and shear strain in metals, nanostructured by the severe plastic deformation. Comparing theoretical and experimental dependencies we regard the scaling parameter of the NET as a fitting parameter and, thus, estimate its value for considered cases. The effect of saturation of material nanofragmentation with increase of the shear strain finds description in the obtained dependencies, with the limiting small grain size being proportional to the scaling parameter of the NET.

\section{Derivation of the Defect-Deformational Kuramoto-Sivashinsky Equation}

Let us consider an elastic solid with nonequilibrium mobile defects (dilatation centers). The energy of defect-strain interaction in the framework of the NET is

$$
H_{d}(\mathbf{r})=-\theta_{d}\left(\xi+l_{d}^{2} \Delta \xi\right)
$$

where $\mathbf{r}=(x, y, z), \theta_{d}$ is the deformation potential of a defect ( $d$ for a dislocation loop, $d=v$ for a vacancy, $\theta_{v}<0$; $d=i$ for an interstitial, $\theta_{i}>0$, and $\theta_{i} \gg\left|\theta_{v}\right|$, see Appendix), $\Delta=$ $\partial^{2} / \partial x^{2}+\partial^{2} / \partial y^{2}+\partial^{2} / \partial z^{2}, \xi(\mathbf{r})=\nabla \mathbf{u}$ is the strain, $\mathbf{u}=\mathbf{u}(\mathbf{r})$ is the medium displacement vector in an isotropic material, and $l_{d}$ is the scaling parameter of the NET. A short derivation of the formula (1) is given in the Appendix.

Using (1), we obtain the elastic force acting on a defect:

$$
\mathbf{F}(\mathbf{r})=-\nabla H(\mathbf{r})=\theta_{d} \nabla\left(\xi+l_{d}^{2} \Delta \xi\right)
$$

where $\nabla=\mathbf{e}_{\mathbf{x}}(\partial / \partial x)+\mathbf{e}_{\mathbf{y}}(\partial / \partial y)+\mathbf{e}_{\mathbf{z}}(\partial / \partial z), \mathbf{e}_{\mathbf{x}}, \mathbf{e}_{\mathbf{y}}$, and $\mathbf{e}_{\mathbf{z}}$ are unit vectors along the $x, y$, and $z$ axes, respectively.

The defect flux consists of the diffusion and straininduced parts:

$$
\mathbf{j}_{d}=-D_{d} \nabla N_{d}+\frac{D_{d}}{k_{B} T} \theta_{d} N_{d} \nabla\left(\xi+l_{d}^{2} \Delta \xi\right),
$$

where $D_{d}$ is the diffusion coefficient of a defect, $k_{B}$ is the Boltzman constant, and $N_{d}\left(\mathrm{~cm}^{-3}\right)$ is the concentration of defects.
From the continuity equation, with allowance for $\mathbf{j}_{d}$, (3), and neglecting the renormalization of the defect diffusivity by the strain, we obtain the equation for $N_{d}$ :

$$
\begin{aligned}
\frac{\partial N_{d}}{\partial t}= & -\frac{1}{\tau} N_{d}+D_{d} \Delta N_{d}-\frac{D_{d} \theta_{d}}{k_{B} T}\left(N_{d} \Delta \xi+\nabla N_{d} \cdot \nabla \xi\right) \\
& -\frac{D_{d} \theta_{d} l_{d}^{2}}{k_{B} T}\left[N_{d} \Delta^{2} \xi+\nabla N_{d} \cdot \nabla(\Delta \xi)\right]+G_{d}
\end{aligned}
$$

where $\tau$ is the defect lifetime and $G_{d}$ is the rate of defect generation.

To close this equation, we need to express the strain $\xi$ through the concentration of defects $N_{d}$. For this end, we use the equation for the displacement vector $\mathbf{u}=\mathbf{u}(\mathbf{r})$ in an isotropic medium from [10]. Taking into account the energy of the strain-defect interaction, (1), where we neglect the term proportional to $l_{d}^{2}$, we modify the equation for $\mathbf{u}$ to the following form:

$$
\frac{\partial^{2} \mathbf{u}}{\partial t^{2}}=c_{t}^{2} \Delta \mathbf{u}+\left(c_{l}^{2}-c_{t}^{2}\right) \nabla(\nabla \mathbf{u})+\frac{\theta_{d}}{\rho} \nabla N_{d},
$$

where $\rho$ is the medium density and $c_{l}$ and $c_{t}$ are the longitudinal and transverse sound velocities, respectively.

Acting with the operator $\nabla$ on both sides of the equation (5), we obtain the equation for the strain:

$$
\frac{\partial^{2} \xi}{\partial t^{2}}=c_{l}^{2} \Delta \xi-\frac{\theta_{d}}{\rho} \Delta N_{d}
$$

In the adiabatic approximation, $\left(\partial^{2} \xi / \partial t^{2}=0\right)$, from (6), we obtain formula for the self-consistent quasistatic strain:

$$
\xi=\frac{\theta_{d}}{\rho c_{l}^{2}} N_{d}
$$

Using (7) in (4), we obtain the following closed equation for defect concentration:

$$
\begin{aligned}
\frac{\partial N_{d}}{\partial t}= & -\frac{1}{\tau} N_{d}+D_{d}\left(1-\frac{N_{d}}{N_{\mathrm{dc}}}\right) \Delta N_{d} \\
& -D_{d} \frac{l_{d}^{2}}{N_{\mathrm{dc}}}\left(\left[N_{d} \Delta^{2} N_{d}+\nabla N_{d} \cdot \nabla\left(\Delta N_{d}\right)\right]\right) \\
& -\frac{D_{d}\left(\nabla N_{d}\right)^{2}}{N_{\mathrm{dc}}}+G_{d},
\end{aligned}
$$

where the critical defect concentration is introduced:

$$
N_{\mathrm{dc}}=\frac{\rho c_{l}^{2} k_{B} T}{\theta_{d}^{2}} .
$$

For point defects, at $\rho c_{l}^{2}=10^{12} \mathrm{erg} * \mathrm{~cm}^{-3}, T=300 \mathrm{~K}, \theta_{d}=$ $10^{2} \mathrm{eV}$ (see Appendix), we have the estimate $N_{d c} \sim 10^{18} \mathrm{~cm}^{-3}$.

The $3 \mathrm{D}$ nonlinear equation (8) is similar to the KS equation $[1,2]$ with the following essential differences. The 
expression in the brackets in the second term on the righthand side of (8) depends on the dynamic variable $N_{d}$ and can change sign with an increase in this variable, while in the KS equation this coefficient is a constant. The coefficient of the dispersion term $\left(\sim \Delta^{2} N_{d}\right)$ also depends on the dynamic variable and is not constant as in the KS equation. Besides, (8) contains an extra term proportional to $\left(\nabla N_{d} \bullet \nabla\left(\Delta N_{d}\right)\right)$.

In the mean field approximation (MFA), the concentration of defects is represented as $N_{d}(\mathbf{r}, t)=N_{d 0}+$ $N_{d 1}(\mathbf{r}, t)$, where $N_{d 0}$ is the spatially uniform concentration and $N_{d 1}(\mathbf{r}, t)$ is the spatially nonuniform component that spontaneously arises above the instability threshold. Under the condition $N_{d 1}(\mathbf{r}, t)<N_{d 0}$, we can neglect the relatively small term $\left(\nabla N_{d} \bullet \nabla\left(\Delta N_{d}\right)\right)$ in the square brackets in (8). Then, introducing the parameter $\varepsilon=N_{d 0} / N_{\mathrm{dc}}$, we obtain from (8) the following equation:

$$
\begin{aligned}
\frac{\partial N_{d 1}}{\partial t}= & -\frac{1}{\tau} N_{d 1}-D_{d}(\varepsilon-1) \Delta N_{d 1} \\
& -D_{d} \varepsilon l_{d}^{2} \Delta^{2} N_{d 1}-\frac{D_{d}}{N_{d c}}\left(\nabla N_{d 1}\right)^{2} .
\end{aligned}
$$

The spatially uniform defect concentration $N_{d 0}$ can be found as a steady state solution of the spatially uniform equation (8):

$$
N_{d 0}=\tau G_{d} .
$$

The spatially uniform defect concentration $N_{d 0}$ (i.e., the parameter $\varepsilon$ ) plays the role of a control parameter of the DD instability.

The DD equation (10) is similar to the stabilized KS equation, which takes into account the term proportional to $\tau^{-1}$. However, a significant difference lies in the fact that (10) contains an additional free parameter $\varepsilon$, which characterizes the excess over DD instability threshold. Note the critical dependence on $\varepsilon$ and the fact that (10) corresponds to the KS equation $[1,2]$ only when $\varepsilon>1$.

\section{The Linear Regime of the DD Instability}

We employ the Fourier-series expansion of the defect concentration:

$$
N_{d}(\mathbf{r}, t)=\sum_{\mathbf{q}} N_{d}(\mathbf{q}, t) \exp (i \mathbf{q r}) .
$$

Here, $N_{d 1}(\mathbf{q})$ is an initial fluctuation amplitudes.

According to (7), the Fourier-series expansion of the strain is

$$
\xi(\mathbf{r}, t)=(\operatorname{div} \mathbf{u})=\frac{\theta_{d}}{\rho c_{l}^{2}} \sum_{\mathbf{q}} N_{d}(\mathbf{q}, t) \exp (i \mathbf{q r}) .
$$

Each pair of Fourier amplitudes $N_{d}(\mathbf{q}, t)$ and $\xi(\mathbf{q}, t)=\left(\theta_{d} /\right.$ $\left.\rho c_{l}^{2}\right) N_{d}(\mathbf{q}, t)$ describes the DD grating with the wave vector q.

For the analysis of the linear regime, we put $N_{d 1}(\mathbf{q}, t)=$ $N_{d 1}(\mathbf{q}) \exp \left(\lambda_{q} t\right)$ in (12). The Fourier transform of the linearized DDKS equation (10) then yields the growth rate of the $\mathrm{DD}$ grating:

$$
\lambda_{q}=D_{d} q^{2}\left[\varepsilon\left(1-l_{d}^{2} q^{2}\right)-1\right]-\frac{1}{\tau} .
$$

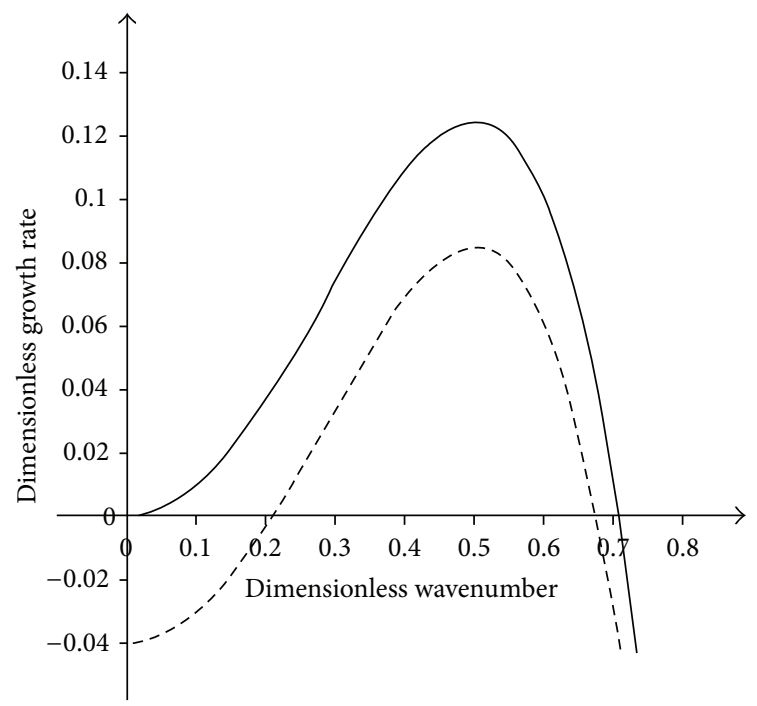

FIGURE 1: Dependence of the dimensionless growth rate of DD Fourier amplitudes, $\tilde{\lambda}(Q)$, on the dimensionless wavenumber $Q$ calculated with formula (15) at $\varepsilon=2, S=0$ (solid), and $S=0.2$ (dashed).

To graphically represent the dependence of the growth rate of DD Fourier amplitudes on the wavenumber, we introduce the dimensionless wavenumber $Q=l_{d} q$, the growth rate $\tilde{\lambda}(Q)=\lambda_{q} /\left(D_{d} l_{d}^{-2}\right)$, and the scaling parameter $S=l_{d} / \sqrt{\tau D_{d}}$. Then, formula (14) acquires the form

$$
\tilde{\lambda}(Q)=Q^{2}\left[\varepsilon\left(1-Q^{2}\right)-1-\frac{S^{2}}{Q^{2}}\right] .
$$

This dependence is shown in Figure 1 at two values of the scaling parameter: $S=0$ and $S=0.2$ (the former corresponds to the case $\tau \rightarrow \infty$ ). It is seen that, when the condition of instability is met (see below), a whole band of DD modes with wavenumbers lying around the dominant (with the maximum growth rate) $\mathrm{DD}$ harmonic with $\mathrm{Q}=\mathrm{Q}_{m}$ (i.e., $q=$ $\left.q_{m}\right)$ becomes unstable. Their amplitudes grow exponentially in time; this leads to the generation of a band of DD Fourier harmonics with $q \sim q_{m}$.

The growth rate $\lambda_{q}$ reaches a maximum value at

$$
q=q_{m}=\frac{\left(1-\varepsilon^{-1}\right)^{1 / 2}}{\sqrt{2} l_{d}} .
$$

The corresponding period of the dominant DD grating with the wave number $q_{m}$ is given by

$$
d_{m}=\frac{2 \pi}{q_{m}}=\frac{2 \pi \sqrt{2} l_{d}}{\left(1-\varepsilon^{-1}\right)^{1 / 2}},
$$

where the control parameter $\varepsilon=N_{d 0} / N_{\mathrm{dc}}$.

The period $d_{m}$ is proportional to the scaling parameter of the NET $l_{d}$, critically increases at $N_{d 0} \rightarrow N_{\mathrm{dc}}$, and reaches 
a constant minimum level at $N_{d 0} / N_{\mathrm{dc}} \gg 1$. For the DD grating with $q=q_{m}$, the maximum growth rate is given by

$$
\lambda_{m}=\frac{D_{d} \varepsilon\left(1-\varepsilon^{-1}\right)^{2}}{4 l_{d}^{2}}-\frac{1}{\tau} .
$$

The growth rate becomes positive $\left(\lambda_{m} \geq 0\right)$, when the condition

$$
\varepsilon \geq \varepsilon_{c} \equiv\left(1+2 \frac{l_{d}^{2}}{\tau D_{d}}\right)+\sqrt{\left(1+2 \frac{l_{d}^{2}}{\tau D_{d}}\right)^{2}-1}
$$

is fulfilled. On the other hand, formula (16) shows that a real value of $q_{m}$ emerges at the condition $\varepsilon>1$. Thus, the critical value of control parameter $\varepsilon_{c}$ is given by the equality in (19). Under the condition $2 l_{d}^{2} / \tau D_{d} \ll 1$, the critical value is $\varepsilon_{c} \approx 1$ and the periodic DD grating is created when the critical defect concentration is exceeded: $N_{d 0}>N_{\mathrm{dc}}(\varepsilon>1)$.

At a constant concentration of defects $\left(N_{d 0}=\right.$ const $)$, the period depends on the temperature $T$ at which the DD instability takes place:

$$
d_{m}=\sqrt{8} \pi l_{d}\left(\frac{T_{c}}{T_{c}-T}\right)^{1 / 2}
$$

where the critical temperature is

$$
T_{c}=\frac{N_{d 0} \theta_{d}^{2}}{k_{B} \rho c_{l}^{2}} .
$$

For point defects, at $\rho c_{l}^{2}=10^{12} \mathrm{erg} * \mathrm{~cm}^{-3}, T=300 \mathrm{~K}, \theta_{d}=$ $10^{2} \mathrm{eV}, N_{d 0} \sim 4 * 10^{18} \mathrm{~cm}^{-3}$, we have the estimate $T_{c} \sim 740 \mathrm{~K}$. Dependencies (17) and (20) are two main results of the linear theory of the DD instability. Comparison of these results with experiment is done in Section 4.

In the linear regime, besides the dominant DD grating with $q=q_{m}$ and the growth rate $\lambda_{m}$, a whole continuum of independent DD gratings with wave vectors $\mathbf{q}$ and growth rates $\lambda_{q}>0$ grows with time and forms the superpositional DD structure (12), (13).

\section{Comparison with Experimental Results}

4.1. The Critical Temperature Dependence of the Grain Size in Metals and Semiconductors, Nanostructured by the SPD. Figure 2 shows the experimental temperature dependence of the grain size in titanium nanostructured by the severe plastic deformation from [11] in comparison with the critical theoretical dependence given by formula (20). The experimental curve is accurately reproduced at $T_{c}=450^{\circ} \mathrm{C}$ and the scaling parameter $l_{d}=11 \mathrm{~nm}$ (for the introduction of this parameter, see Appendix).

It is interesting to note that, in semiconductors, nanostructured by severe plastic deformation, the mean grain size is of an order of magnitude less than that in metals $(24 \mathrm{~nm}$ in $\mathrm{Ge}$ and $17 \mathrm{~nm}$ in $\mathrm{Si}$ [11]). Based on results of the present work one may suppose that the scaling parameter of the NET- $l_{d}$ in semiconductors is of order of $1 \mathrm{~nm}$.

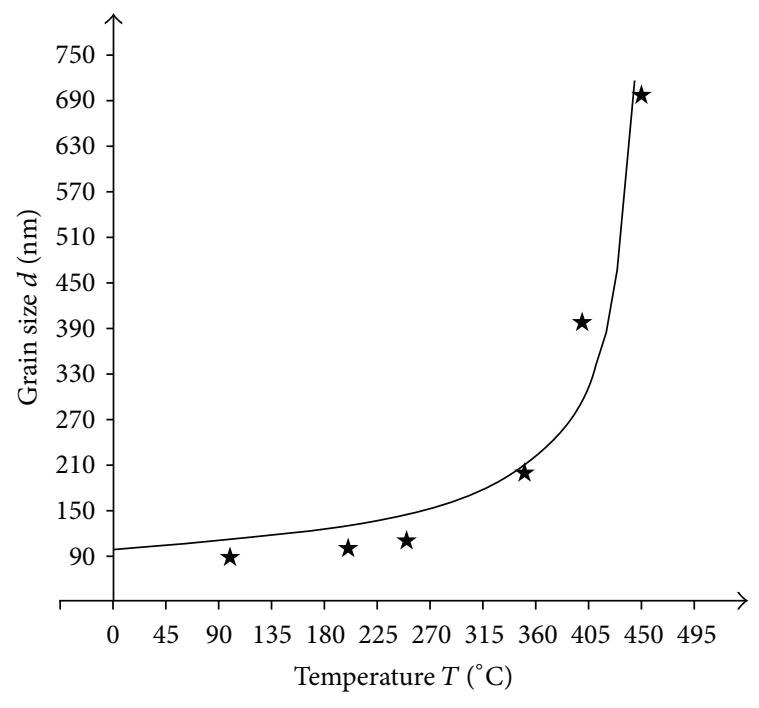

FIGURE 2: Experimental temperature dependence of the grain size in titanium nanostructured by the severe plastic deformation [11] (stars). The curve is drawn with the use of formula (20) at $l_{d}=$ $11 \mathrm{~nm}, T_{c}=450^{\circ} \mathrm{C}$.

4.2. The Critical Shear Stress Dependence of the Grain Size in Metals and Semiconductors, Nanostructured by the SPD and the Saturation of Nanofragmentation. Figure 3 shows the experimental shear strain dependence of the grain size in nickel nanostructured by the SPD at room temperature [12]. To model this dependency, we used formula (17), where we set

$$
\varepsilon=\beta \cdot \gamma
$$

where $\gamma$ is shear strain in the material induced during the SPD and $\beta$ is the proportionality constant. We note that the linear dependence of the defect (vacancy) concentration generated by the SPD on the shear strain is confirmed experimentally (see, e.g., [13]).

A good fit of the experimental dependence is obtained at $\beta=0.435$ and the value of the scaling parameter $l_{d}=43 \mathrm{~nm}$ (Figure 3).

It is seen that the theoretical curve describes two salient features of the experimental dependence: critical increase of the grain size with approaching the critical value of the shear strain $\left(\gamma \rightarrow \gamma_{c}=2.3\right)$ and the saturation of the grain size decrease at $\gamma \rightarrow \infty$.

One more correspondence between the theory and experiment is obtained if one compares the point defect concentration $N_{d 0}$, taking part in the DD instability, with the experimentally determined defect concentration. Since $\varepsilon=N_{d 0} / N_{\mathrm{dc}}$ and $N_{\mathrm{dc}} \sim 10^{18} \mathrm{~cm}^{-3}$ (see (9), from (22) one has the estimate of point defect (vacancy) concentration: $N_{d 0} \sim$ $0.435 \gamma * 10^{18} \mathrm{~cm}^{-3} \sim 1.7 * 10^{18}-1.3 * 10^{19} \mathrm{~cm}^{-3}$ within the interval $4 \leq \gamma \leq 30$, shown in Figure 3. This estimate of vacancy concentration is in accordance with characteristic experimentally measured values for $\mathrm{Cu}$ and $\mathrm{Ni}$ achieved with SPD (see $[13,14])$. 


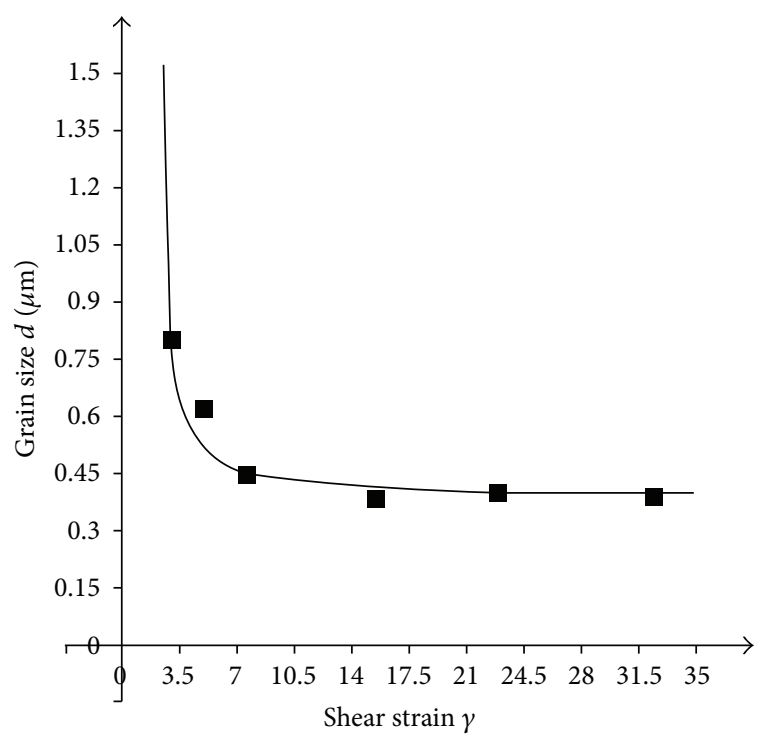

Figure 3: Experimental dependence of the grain size in nickel nanostructured by the SPD [12] (squares) on the shear strain $\gamma$. The curve is drawn according to formula (17) at $l_{d}=43 \mathrm{~nm}$ and $\varepsilon=0.435 \gamma$.

\section{The Outline of a New DD Mechanism of Nanostructuring of Solids under the Severe Plastic Deformation and Conclusion}

Based on results obtained in this work, we propose the following novel scenario of nanostructuring of solids upon the severe plastic deformation. Let us consider a polycrystalline solid with the initial grain size $L$. Consider one of such grains. The SPD process leads to the generation of the dense field of defects with a mean concentration $N_{d 0}$ in the bulk of this grain. If $N_{d 0} \geq N_{\mathrm{dc}}$, then the threshold DD selforganization of defects occurs in the volume of the grain leading to the formation of the $3 \mathrm{D} \mathrm{DD}$ structure with the period $d$, which is the function of $N_{d 0}$ (see (17)). The DD selforganization process is governed by 3D DDKS equation (10). Inspecting numerical solutions of similar 2D DDKS [5], one may expect the formation in the considered $3 \mathrm{D}$ isotropic solid of a 3D cellular hexagonal DD structure. After the DD selforganization, the initial large grain of size $L$ is fragmented into small grains of size $d<L$. Boundaries of small grains are formed by piles of defects with the concentration $N_{d 1}$. At this stage, the defect field consists of two parts: defects with concentration $N_{d 0}$ in the bulk of the small grains and defects with concentration $N_{d 1}$ accumulated in the boundaries of small grains $\left(N_{d 1}<N_{d 0}\right)$. The DD walls (small grain boundaries) are in a dynamic equilibrium with the defect concentration field $N_{d 0}$. An increase of $N_{d 0}$ leads, according to (17), to decrease of $d$ (see Figure 2). Thus, the continuation of the SPD process, accompanied by the shear strain $\gamma$ (and defect concentration) increase, leads to further fragmentation of grains occurring via grain boundaries rearrangement. From Figure 2 it is seen that, at large $\gamma$, the saturation of fragmentation occurs at the minimal grain size $d_{\min }=2 \pi \sqrt{2} l_{d}$.
This picture of successive fragmentation of an initial large grain into small ones is observed in experiment together with the nanofragmentation saturation effect (see [12]).

When $N_{d 1}>N_{\mathrm{dcl}}$, where $N_{\mathrm{dcl}}$ is the threshold defect concentration of dislocation nucleation, the formation of dislocation loops begins at DD grain boundaries. At this stage of the DD self-organization, grain boundaries decouple from the bulk, spatially uniform defect concentration field $N_{d 0}$, and the structure of nanofragments with a small size $d$, achieved at this point, is written permanently and irreversibly inside the initial large grain of size $L$. The grain boundaries in this structure consist of dislocation pileups. If $d<d_{\min }$, we return to the initial point of our consideration with a grain of size $d$ playing the role of starting large grain $L$. If the process of the SPD is resumed, it will lead to further fragmentation of the grain with size $d$ until the saturation of fragmentation occurs.

In conclusion, the novel DDKS equation for nonequilibrium defect concentration is derived, and its linear solutions are shown to reproduce salient experimental dependencies describing the nanofragmentation of solids during the SPD. The new DD mechanism of nanofragmentation based on the notion of nonequilibrium defects generation and selforganization during the SPD is proposed.

\section{Appendix}

\section{The Derivation of Energy of Defect-Strain Interaction in the Framework of the Nonlocal Elasticity Theory}

We define the energy of the defect-strain interaction as a work done under creation of a defect (dilatation center) in the stressed material. In an isotropic solid, this work is

$$
H_{d}(\mathbf{r})=-\sigma(\mathbf{r}) \Delta V_{d},
$$

where $\sigma(\mathbf{r})$ is the nonlocal stress at point $\mathbf{r}$ and $\Delta V_{d}$ is the change of the sample volume under creation of the defect in its bulk $\left(\Delta V_{v}<0\right.$ for vacancies, $\Delta V_{i}>0$ for interstitials, and $\left.\Delta V_{i} \gg\left|\Delta V_{v}\right|\right)$.

The equation for the nonlocal stress in the considered case is

$$
\left(1-l_{d}^{2} \Delta \sigma\right)=K \xi
$$

where $K$ is the bulk elasticity modulus. The (A.2) follows from (4) of [15] in the case of isotropic solid.

Solving (A.2) with the use of perturbation theory, we obtain the nonlocal stress:

$$
\sigma(\mathbf{r})=K \xi(\mathbf{r})+l_{d}^{2} K \Delta \xi(\mathbf{r}) .
$$

Substituting (A.3) in (A.1), we obtain formula for defectstrain interaction:

$$
H_{d}(\mathbf{r})=-\sigma(\mathbf{r}) \Delta V_{d} \sigma(\mathbf{r})=-\theta_{d}\left(\xi(\mathbf{r})+l_{d}^{2} \Delta \xi(\mathbf{r})\right),
$$

where $\theta_{d}=K \Delta V_{d}$ is the deformation potential for a defect. For point defects: $\theta_{v}<0, \theta_{i}>0$ and $\theta_{i} \gg\left|\theta_{v}\right|$. At 
$K=10^{12} \operatorname{erg} * \mathrm{~cm}^{-3},\left|\Delta V_{d}\right|=a^{3} \sim 10^{-22} \mathrm{~cm}^{3}$ ( $a$ is the crystal lattice parameter), we have the estimate $\theta_{d} \sim 10^{2} \mathrm{eV}(d=v, i)$.

The case of small (nanometer size) dislocation loops as strongly anisotropic dilatation centers needs separate consideration and is not addressed here.

\section{References}

[1] Y. Kuramoto, Chemical Oscillations, Waves and Turbulence, Springer, Berlin, Germany, 1984.

[2] G. I. Sivashinsky, "Instabilities, pattern formation, and turbulence in flames," Annual Review of Fluid Mechanics, vol. 15, pp. 179-199, 1983.

[3] J. M. Garcia, L. Vazquez, R. Cuerno, J. A. Garcia, M. Castro, and R. Gago, "Self-organized surface nanopatterning by ion beam sputtering," in Lecture Notes on Nanoscale Science and Technology 5, Z. Wang, Ed., pp. 323-398, Springer, Heidelberg, Germany, 2009.

[4] A. G. Limonov, "Numeric simulation of the formation of hexagonal nanoscale structure arrays in Anodic Aluminum Oxide," Mathematical Models and Computer Simulations, vol. 3, no. 2, pp. 149-157, 2011.

[5] V. I. Emel'yanov, "Mechanisms of laser-induced self-organization of nano- and microstructures of surface relief in air and in liquid environment," in Laser Ablation in Liquids, Principles and Applications in the Preparation of Nanomaterials, G. Yang, Ed., chapter 1, pp. 1-109, Pan Stanford, Singapore, 2012.

[6] V. I. Emel'yanov and I. M. Panin, "Formation of nanometer ordered defect-deformational structures induced by energy fluxes in solids," Physics of the Solid State, vol. 39, no. 11, pp. 18151820, 1997.

[7] K. Arakawa, K. Ono, M. Isshiki, K. Mimura, M. Uchikoshi, and H. Mori, "Observation of the one-dimensional diffusion of nanometer-sized dislocation loops," Science, vol. 318, no. 5852, pp. 956-959, 2007.

[8] I. A. Kunin, Theory of Elastic Media with Microstructure, Springer, New York, NY, USA, 1982.

[9] A. C. Eringen, Nonlocal Continuum Field Theories, Springer, New York, NY, USA, 2002.

[10] L. D. Landau and E. M. Lifshitz, Theory of Elasticity, Pergamon, New York, NY, USA, 1986.

[11] R. Z. Valiev, R. K. Islamgaliev, and I. V. Alexandrov, "Bulk nanostructured materials from severe plastic deformation," Progress in Materials Science, vol. 45, no. 2, pp. 103-189, 2000.

[12] R. Pippan, S. Scheriau, A. Taylor, M. Hafok, A. Hohenwarter, and A. Bachmaier, "Saturation of fragmentation during severe plastic deformation," Annual Review of Materials Research, vol. 40, pp. 319-343, 2010.

[13] E. Schafler, G. Steiner, E. Korznikova, E. Kerber, and M. J. Zehetbauer, "Lattice defect investigation of ECAP-Cu by means of X-ray line profile analysis, calorimetry and electrical resistometry," Materials Science and Engineering A, vol. 410411, pp. 169-173, 2005.

[14] D. Setman, E. Schafler, E. Korznikova, and M. J. Zehetbauer, "The presence and nature of vacancy type defects in nanometals detained by severe plastic deformation," Materials Science and Engineering A, vol. 493, no. 1-2, pp. 116-122, 2008.

[15] Y. Y. Zhang, C. M. Wang, W. H. Duan, Y. Xiang, and Z. Zong, "Assessment of continuum mechanics models in predicting buckling strains of single-walled carbon nanotubes," Nanotechnology, vol. 20, no. 39, Article ID 395707, 2009. 

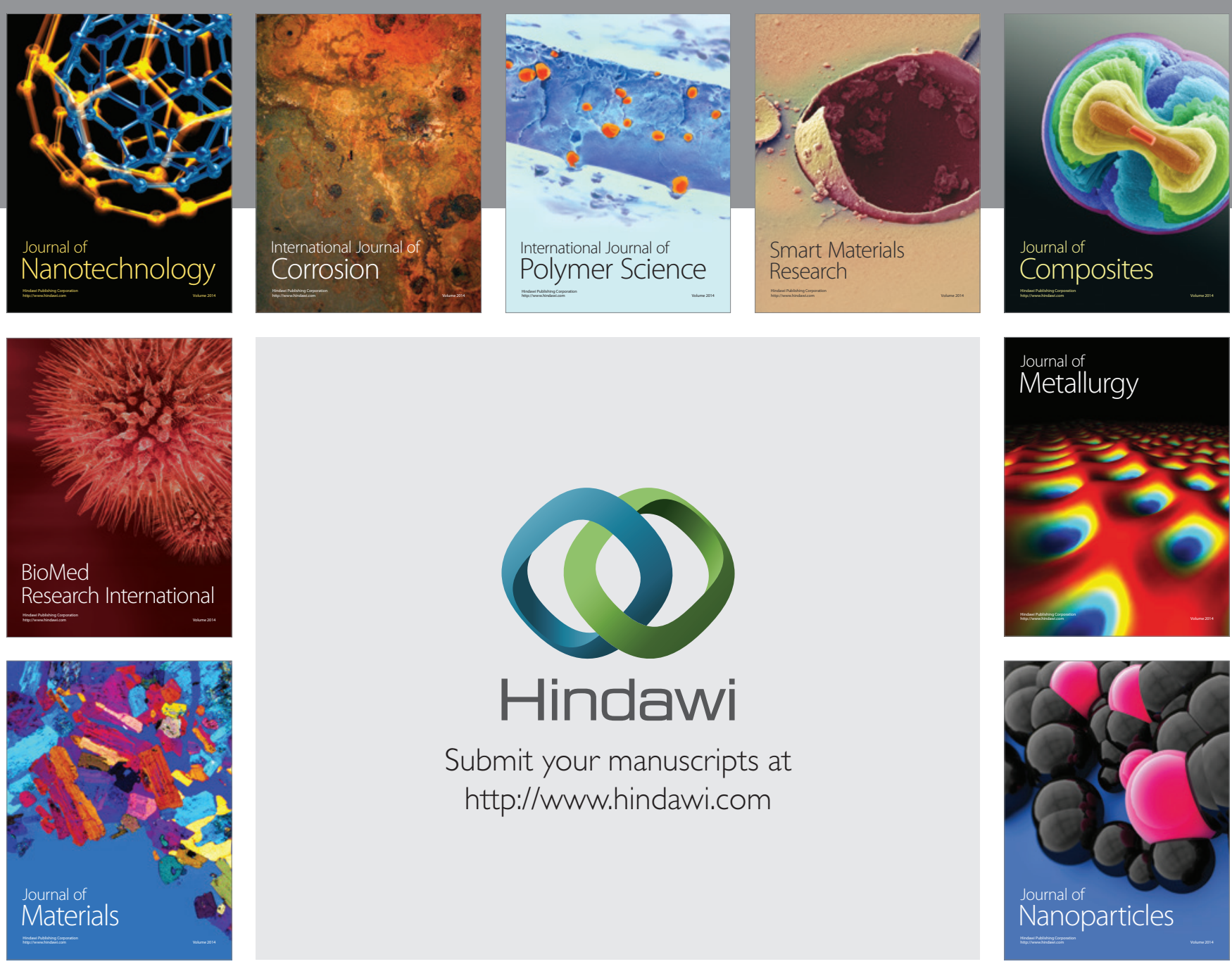

Submit your manuscripts at http://www.hindawi.com
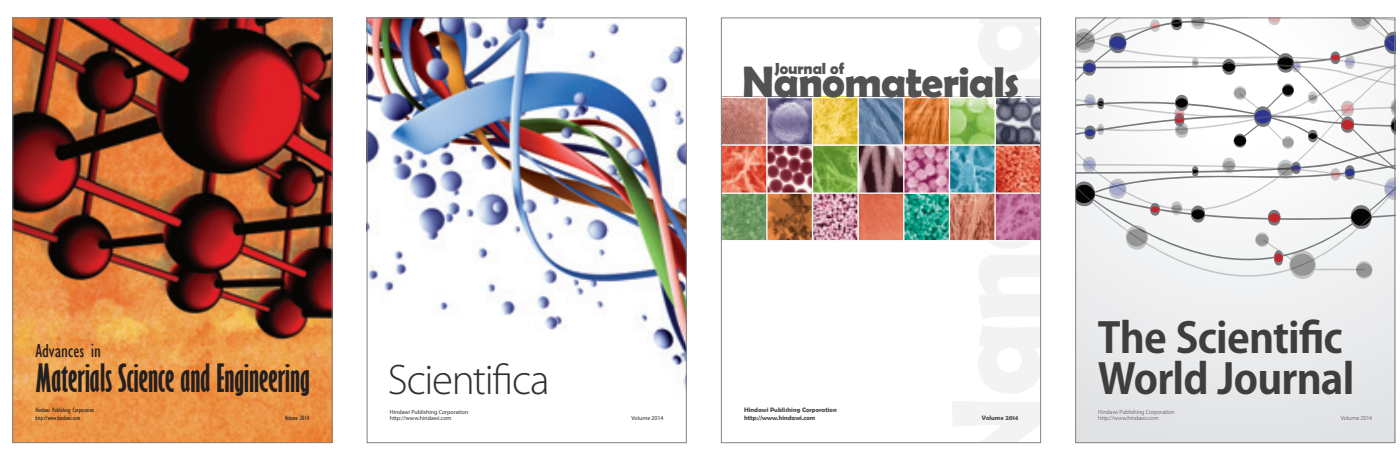

\section{The Scientific World Journal}
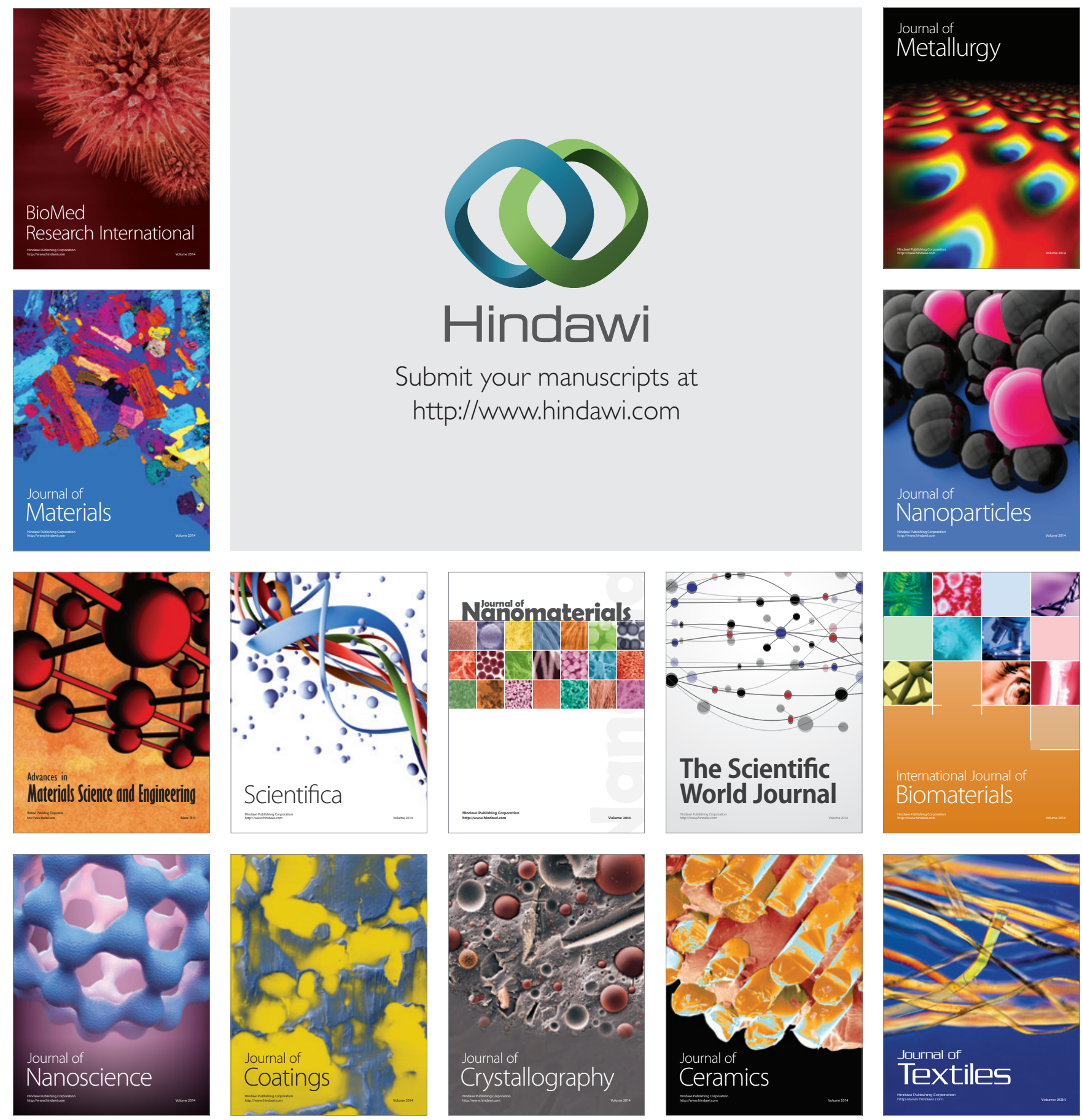